\title{
Study of amniotic fluid index and its pregnancy outcome
}

\author{
Manisha M. Parmar'1, Sandeep M. Parmar ${ }^{2 *}$ \\ ${ }^{1}$ Department of Obstetrics and Gynecology, P. D. U. Medical College, Rajkot, Gujarat, India
}

${ }^{2}$ Consultant Gynecologist, Parul Maternity Home, Vadodara, Gujarat, India

Received: 14 November 2019

Accepted: 18 November 2019

\section{*Correspondence:}

Dr. Sandeep M. Parmar,

E-mail: sandeepdipa12013@gmail.com

Copyright: $\odot$ the author(s), publisher and licensee Medip Academy. This is an open-access article distributed under the terms of the Creative Commons Attribution Non-Commercial License, which permits unrestricted non-commercial use, distribution, and reproduction in any medium, provided the original work is properly cited.

\begin{abstract}
Background: Amniotic fluid is vital to the well-being of the fetus. Severe oligohydramnios and polyhydramnios are associated with increased maternal morbidity and perinatal morbidity and mortality.

Methods: This was prospective observational study conducted at tertiary teaching institute from July 2012 to July 2013. Total 200 patients were included in the study. On the basis of amniotic fluid index (AFI), patients were categorized in 3 groups, Normal AFI $(8-24 \mathrm{~cm}$ ), oligohydramnios (AFI $\langle 5 \mathrm{~cm})$ and polyhydramnios (AFI $\rangle=25 \mathrm{~cm}$ ). Results were analysed in the form of incidence, mode of delivery and perinatal outcome which includes preterm, low birth weight, still births, NICU admissions and neonatal deaths in all the 3 groups.

Results: Out of 200 patients, there was 150 cases of normal AFI, 39 cases of oligohydramnios and 11 cases of polyhydramnios. Incidence of oligohydramnios was $4.1 \%$ and polyhydramnios was $1.1 \%$. PIH was the most common etiological factor found in oligohydramnios $(30.7 \%)$ and in polyhydramnios congenital anomalies (36.3\%) followed by idiopathic cause (27.2\%) was most common. Incidence of caesarean section was $58.9 \%$ in oligohydarmnios and $17.3 \%$ in normal AFI group. Incidence of NICU admission was $25.6 \%$ in oligohydramnios and $50 \%$ in polyhydramnios group in comparison to $9.3 \%$ in normal AFI group.

Conclusions: Amniotic fluid index is an important part of antepartum fetal surveillance. Abnormalities of AFI are associated with high perinatal morbidity and mortality and maternal morbidity.
\end{abstract}

Keywords: Amniotic fluid index, Oligohydramnios, Polyhydramnios

\section{INTRODUCTION}

Amniotic fluid is vital to the well-being of the fetus. It cushions the fetus from injury, helps to prevent compression of the umbilical cord, and allows room for it to move and grow.

In addition, its bacteriostatic action helps to prevent infection of the intra-amniotic environment. The quantity of amniotic fluid at any time in gestation is the product of water exchange between the mother, fetus, and placenta, and is maintained within a relatively narrow range. Disorders of this regulatory process can lead to either polyhydramnios or oligohydramnios, in which too much or too little fluid exists, respectively. These disorders may result from abnormal fetal or maternal conditions and, conversely, may be responsible for alterations of fetal well-being as well. With the advent of real-time ultrasonography, assessment of amniotic fluid has been possible, resulting in earlier recognition of abnormal conditions and possible intervention.

Since, these disorders of liquor amnii has a significant impact on pregnancy and fetus, it prompted us to carry out this study with sincere efforts to find out its effect on pregnancy outcome. ${ }^{1,2}$ 
The objective of this study were;

- To find out incidence of oligohydramnios and polyhydramnios in our setup

- To study possible etiological factors for abnormal AFI

- To find out mode of delivery

- To study perinatal outcome in the form of live birth, still birth, NICU admission and neonatal deaths.

\section{METHODS}

The present study is a prospective observational study done at tertiary teaching institute. From July 2012 to July 2013. During this study period, 200 patients were selected at our tertiary care centre. A detailed history was taken and thorough clinical examination was done. All routine antenatal investigations were done. AFI was measured by four quadrant technique by USG and patients were grouped as normal (AFI 8-24 cm), oligohydramnios (AFI $<5 \mathrm{~cm}$ ) and polyhydramnios (AFI $>25 \mathrm{~cm}$ ). Cases were managed accordingly. Data were analysed in terms of incidence, etiology, mode of delivery, and perinatal outcome in the form of live births, still births, NICU admissions and neonatal mortality in all the three groups. The rate of each outcome was calculated in all the three groups and then the two groups that is oligohydramnios and polyhydramnios are compared with normal AFI group with chi square test. $\mathrm{p}$ value $<0.05$ was considered significant.

\section{Inclusion criteria}

- Antenatal patients in third trimester.

\section{Exclusion criteria}

- Antenatal patients having premature rupture of membranes.

\section{RESULTS}

Total 7948 deliveries were conducted during 1 year, of which 451 cases of abnormal AFI in our institute, so the Incidence of oligohydramnios were $4.1 \%$ and polyhydramnios were $1.1 \%$.

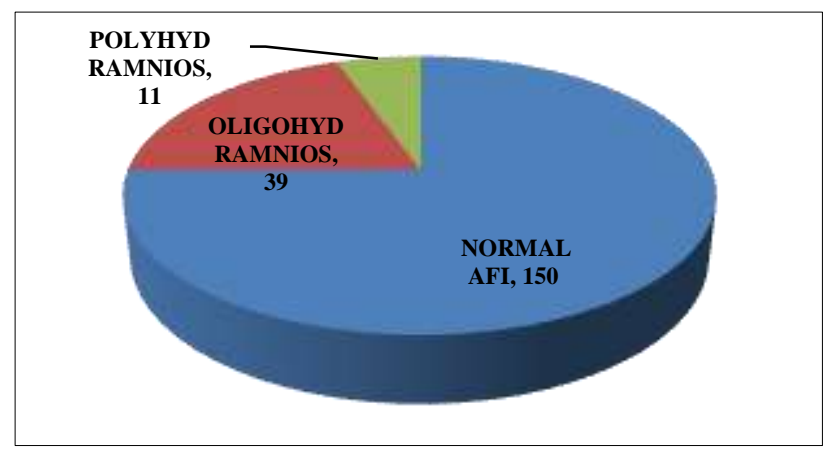

Figure 1: Incidence of oligohydramnios and polyhydramnios.

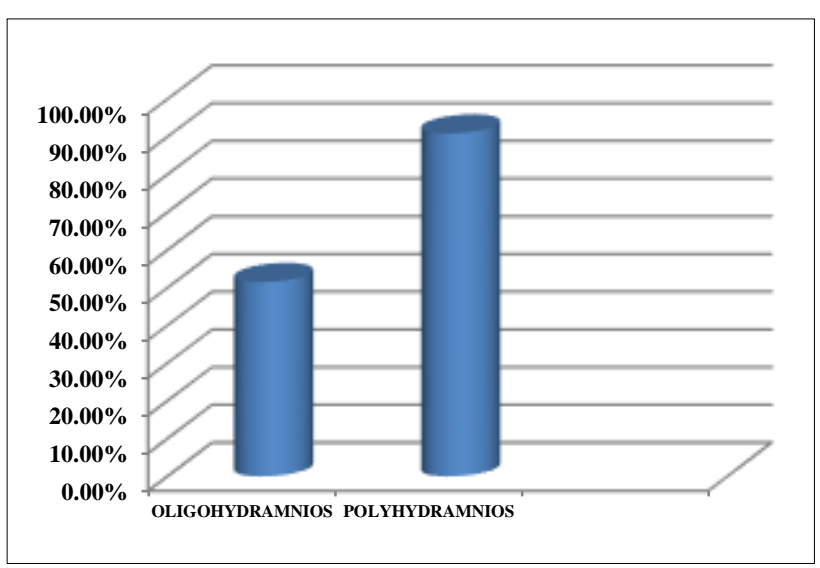

Figure 2: Preterm labour and its association with AFI.

Table 1: Associated conditions (etiology).

\begin{tabular}{|llll|}
\hline Associated conditions & Normal AFI No. (\%) & Oligohydramnios No. (\%) & Polyhydramnios No. (\% \\
\hline Pregnancy induced hypertension & $21(14 \%)$ & $12(30.7 \%)$ & $1(9 \%)$ \\
\hline Intrauterine growth restriction & $11(7.3 \%)$ & $10(25.6 \%)$ & - \\
\hline Postdatism & $10(6.6 \%)$ & $6(15.4 \%)$ & - \\
\hline Congenital anomaly & $7(4.6 \%)$ & - & $4(36.3 \%)$ \\
\hline Fever & $1(0.6 \%)$ & $4(10.2 \%)$ & - \\
\hline Diabetes & $3(2 \%)$ & - & $2(18.1 \%)$ \\
\hline RH incompatibility & - & - & $1(9 \%)$ \\
\hline Idiopathic & - & $7(17.9 \%)$ & $3(27.2 \%)$ \\
\hline
\end{tabular}

Total 200 patients were included in the study from July 2012 to July 2013. Out of 200 patients, 150 patients were with normal AFI, 39 patients were with oligohydramnios and 11 patients were with polyhydramnios (Figure 1). 
In oligohydramnios, main etiological factor found was PIH $(30.7 \%)$, whereas in polyhydramnios, congenital anomalies $(36.3 \%)$ were the most common associated factor followed by idiopathic etiology (27.2\%) (Table 1).

Preterm delivery seen in 20 cases (51.2\%) in oligohydramnios, 10 cases $(90.9 \%)$ in polyhydramnios and 45 cases $(30 \%)$ in normal AFI group (Figure 2 ).

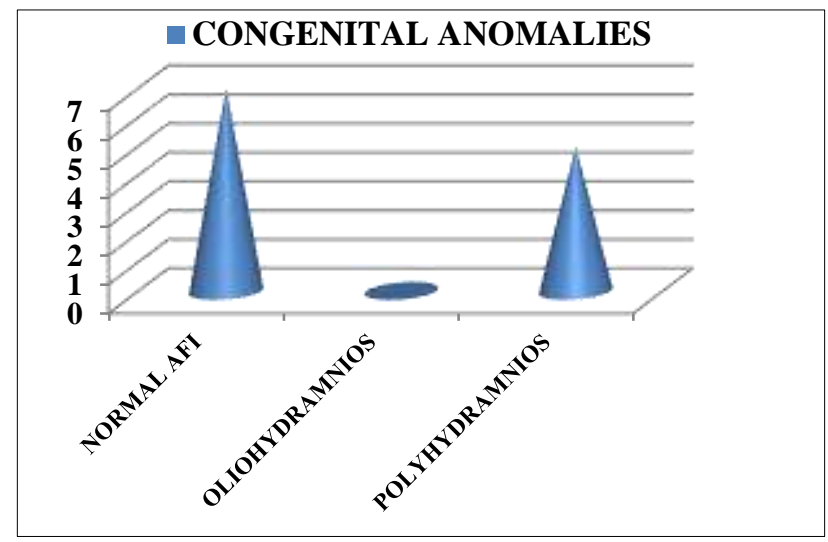

Figure 3: Incidence of congenital anomalies.

In present series of cases, congenital anomalies were more common in patients of polyhydramnios $(45.4 \%)$ as compared to normal AFI (4.6\%). No association was found in patients of normal AFI and oligohydramnios with congenital anomalies in our study (Figure 3).

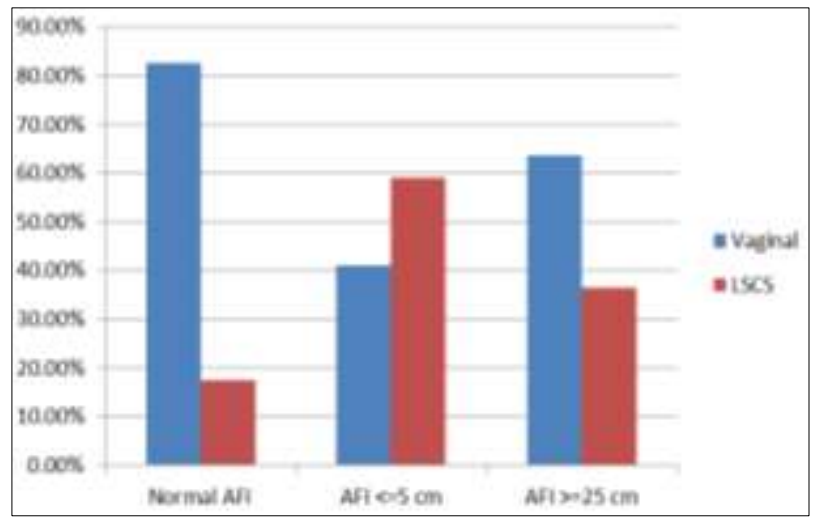

Figure 4: AFI and maternal outcome of labour.

Caesarean section was done in 23 cases $(58.9 \%)$ in oligohydarmnios, 4 cases $(36.3 \%)$ in polyhydramnios in comparision to 26 cases $(17.3 \%)$ in normal AFI group (Figure 4).

Majority of LSCS were done for fetal distress, preeclampsia and malpresentation. Fetal distress in the form of meconium stained liquor, fetal bradycardia or tachycardia were more in patients with abnormal liquor that is $91.3 \%$ in oligohydramnios and $50 \%$ in polyhydramnios as compared to normal liquor (46.1\%).

It can be be due to cord compression or IUGR in patients with oligohydramnios. In polyhydramnios group, caesarean section was done in one patient for prolonged labour and in another one patient for cord prolapse (Table 2).

Table 2: Indications of caesarean section.

\begin{tabular}{|llll|}
\hline Indications & Normal AFI & Oligohydramnios & Polyhydramnios \\
\hline Fetal distress & $12(46.1 \%)$ & $21(91.3 \%)$ & $2(50 \%)$ \\
\hline Pre-eclampsia & $4(15.3 \%)$ & $1(4.3 \%)$ & - \\
\hline IUGR & - & $1(4.3 \%)$ & $-^{-}$ \\
\hline Malpresentation & $5(19.2 \%)$ & - & - \\
\hline Prolonged labour & - & - & $1(25 \%)$ \\
\hline Cephalopelvic disproportion (CPD) & $1(3.8 \%)$ & - & - \\
\hline Antepartum haemorrhage (APH) & $4(15.3 \%)$ & - & - \\
\hline Cord prolapse & - & - & $1(25 \%)$ \\
\hline Total & $\mathbf{2 6}$ & $\mathbf{2 3}$ & $\mathbf{4}$ \\
\hline
\end{tabular}

Table 3: Perinatal outcome.

\begin{tabular}{|llll|}
\hline $\begin{array}{l}\text { Perinatal } \\
\text { outcome }\end{array}$ & Live birth & Still birth & $\begin{array}{l}\text { Neonatal } \\
\text { death }\end{array}$ \\
\hline Normal AFI & 147 & 3 & 5 \\
\hline Oligohydramnios & 34 & 5 & 4 \\
\hline Polyhydramnios & 9 & 2 & 2 \\
\hline
\end{tabular}

A total 5 neonatal deaths occurred in normal AFI group, 4 in oligohydramnios group and 2 in polyhydramnios group (Table 3).

A total $66.6 \%$ babies in oligohydramnios group and $81.8 \%$ in polyhydramnios group were of LBW $(<2.5 \mathrm{~kg})$ (Table 4). 
APGAR score was low $(<7$ at $5 \mathrm{~min}$ ) in $20.5 \%$ of patients with oligohydramnios and $27.2 \%$ in polyhydramnios in comparison to $1.3 \%$ in normal AFI group (Table 4). NICU admission seen in $25.6 \%$ in oligohydramnios and $50 \%$ in polyhydramnios group in comparison to $9.3 \%$ in normal AFI group (Table 4).

Table 4: Perinatal morbidity.

\begin{tabular}{|llllllllll|}
\hline Outcome & \multicolumn{2}{c}{ Low birth weight } & \multicolumn{2}{l|}{ Low A PGAR } & \multicolumn{3}{c|}{ NICU admission } \\
\hline AFI & Normal & Oligo & Poly & Normal & Oligo & Poly & Normal & Oligo & Poly \\
\hline Present study & $39.3 \%$ & $66.6 \%$ & $81.8 \%$ & $1.39 \%$ & $20.5 \%$ & $27.2 \%$ & $9.3 \%$ & $25.6 \%$ & $50 \%$ \\
\hline
\end{tabular}

No maternal deaths occurred in this study.

\section{DISCUSSION}

In present study, incidence of oligohydramnios was $4.1 \%$ and polyhydramnios was $1.1 \%$. Casey $\mathrm{B}$ et al, study on oligohydramnios showed $2.3 \%$ and Fawad A et al study on polyhydramnios showed $2 \%$ incidence. . $^{3,4}$

In present study, in majority of cases, the cause of oligohydramnios was $\mathrm{PIH}$ accounting for $30.7 \%$ cases, and idiopathic $17.9 \%$ cases. The most common cause for polyhydramnios was congenital anomalies (36.3\%) followed by idiopathic cause (27.2\%). Study by Akhter et al and Rajsriya et al, on oligohydramnios also showed the results comparable with our study while the study on polyhydramnios by Queenan and gandow and Fawad A et al showed that Idiopathic cause was more common $(56 \%$ and $50 \%$ ) followed by congenital anomalies (20\% and $28 \%){ }^{4-7}$

In the patients with normal AFI, $45(30 \%)$ patients were delivered preterm. $10 \quad(90.9 \%)$ patients with polyhydramnios were delivered preterm. The incidence is much higher than study by Chen $\mathrm{KC}$ et al, which showed $25 \%$ preterm deliveries in polyhydramnios. ${ }^{8}$

Preterm delivery was seen in $20(51.2 \%)$ patients with oligohydramnios which is comparable with the study by Garmel and co-workers showing oligohydramnios prior to 37 weeks due to PIH, IUGR had a threefold increase in preterm births. $^{9}$

In present series of cases, congenital anomalies were more common in patients of polyhydramnios $(45.4 \%)$ as compared to normal AFI (4.6\%). Study done by Kale A et al, on polyhydramnios showed a significant large association between polyhydramnios and congenital anomalies. $^{10}$

In present study, out of 39 patients of oligo $23(58.9 \%)$ cases underwent LSCS, $16(41 \%)$ cases had vaginal delivery. Out of 23 cases who underwent LSCS 21 $(91.3 \%)$ cases were due to fetal distress. Out of 11 cases of polyhydramnios $4(36.3 \%)$ had LSCS and 7 (63.6\%) had vaginal delivery.
Rate of caesarean section was found to be significantly higher in oligohydarmnios $(58.9 \%)$ in comparison to normal AFI group (17.3\%). No association between polyhydramnios and higher rate of LSCS can be found in our study in comparison to normal AFI group.

Incidence of Low birth weight was higher in patients with oligohydramnios and polyhydramnios that is $66.6 \%$ and $81.8 \%$ respectively as compared to normal AFI (39.3\%). Akhter et al, showed $40 \%$ LBW babies in patients with normal liquor while $60 \%$ babies with LBW in patients with oligohydramnios. ${ }^{5}$ Chen $\mathrm{KC}$ et al, showed $22.6 \%$ babies with LBW in polyhydramnios compared to $6.1 \%$ in patients with normal liquor. ${ }^{8}$

Low APGAR score was significantly higher in patients with oligohydramnios and polyhydramnios that is $20.5 \%$ and $27.2 \%$ respectively compared to normal liquor (1.3\%). In a study on oligohydramnios by Jandial C et al, showed $12 \%$ babies with low APGAR score while Chen $\mathrm{KC}$ et al study on polyhydramnios showed $11.1 \%$ babies with Low APGAR score which is also comparable with our study. ${ }^{8,11}$

There was $25.6 \%$ and $50 \%$ NICU admissions in oligohydramnios and polyhydramnios respectively as compared to $9.3 \%$ in patients with normal AFI. Sadovsky et al, study on oligohydramnios showed $17 \%$ NICU admissions and Chen $\mathrm{KC}$ et al study on polyhydramnios showed $18.6 \%$ NICU admissions..$^{8,12}$

Out of 39 cases of oligo, still birth was 5 and neonatal deaths occurred in 4 while out of 11 cases of polyhydramnios 2 were still born and 2 were neonatal deaths.

\section{CONCLUSION}

Abnormalities of AFI both reduced and excess liquor are associated with high maternal morbidity and perinatal morbidity and mortality. Ultrasonography proved to be an important tool for early and accurate diagnosis of oligo and polyhydramnios and also to rule out congenital malformations and hence to improve maternal and fetal outcome. Oligohydramnios is frequent finding in pregnancies involving IUGR, $\mathrm{PIH}$ and post-datism. Polyhydramnios is associated with congenital malformations. AFI abnormalities demands intensive 
fetal surveillance and proper antepartum and intrapartum care. Timely decision for intervention is helpful in reducing perinatal morbidity and mortality.

Funding: No funding sources

Conflict of interest: None declared

Ethical approval: The study was approved by the Institutional Ethics Committee

\section{REFERENCES}

1. American College of Obstetricians and Gynecologists: Amnioinfusion does not prevent meconium aspiration syndrome. Obstet Gynecol. 2006; 108:1053.

2. Deka D, Malhotra B. Role of maternal oral hydration in increasing amniotic fluid volume in pregnant women with oligohydramnios. Int $\mathbf{J}$ Gynaecol. 2001;73:155.

3. Casey B, Mcintire D, Bloom S. Pregnancies outcomes after antepartum diagnosis of oligohydramnios at or beyond 34 weeks' gestation. Am J Obstet Gynecol. 2000;182:909-12.

4. Fawad A, Danish N. Frequency, causes and outcome of polyhydramnios. Gomal J Med Sci. 2008;6:2.

5. Akhter H, Guha K, Daisy KP. Amniotic fluid Index in high risk pregnancies and pregnancy outcome. Dinajpur Med Col. 2010;3(1):1-5.
6. Sriya R, Singhai S, Rajan M. Perinatal outcome in patients with amniotic fluid index $<5 \mathrm{~cm}$. J Obstet Gynaecol India. 2001;51(5):98-100.

7. Queenan JT. Polyhydramnios and oligohydramnios. Contemp Obstet Gynecol. 1991;36:60.

8. Chen KC, Liou JD, Hung TH, Kuo DM, Hsu JJ, Hsieh CC, et al. Perinatal outcomes of polyhydramnios without associated congenital fetal anomalies after the gestational age of 20 weeks. Chang Gung Med J. 2005;28(4):222-8.

9. Garmel SH, Chelmow D, Sandra JS, Roan JT, D'Alton ME. Oligohydramnios and the appropriately grown fetus. Am J Perinatol. 1997;14(06):359-63.

10. Kale A, Akdeniz N, Erdemoolu M, Yalankaya A, Yayla M. Retrospective analysis of polyhydramnios cases. Perinat J. 2005;13:23-9.

11. Jandial C, Gupta S, Sharma S, Gupta M. Perinatal outcome after antepartum diagnosis of oligohydramnios at or beyond 34 weeks of gestation. JK Sci. 2007;9(4):213-4.

12. Sadovsky YO, Christensen MW, Scheerer LO, Crombleholme WR. Cord-containing amniotic fluid pocket: a useful measurement in the management of oligohydramnios. Obstet Gynecol. 1992;80(5):775-7.

Cite this article as: Parmar MM, Parmar SM. Study of amniotic fluid index and its pregnancy outcome. Int J Reprod Contracept Obstet Gynecol 2019;8:4727-31. 